\title{
Unfallfreier Chefwechsel
}

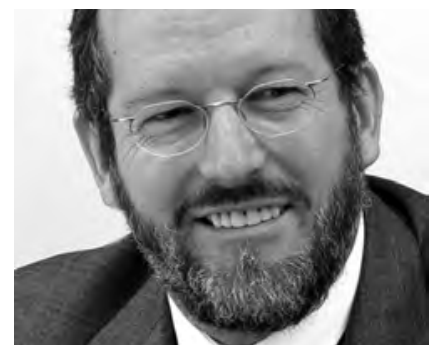

VON MARTIN BECK Prof. Martin Beck, Großhandelskaufmann und Diplombetriebswirt (FH), war Geschäftsführer im Diakonischen Werk Württemberg, Gründer und Geschäftsführer einer Beratungsgesellschaft und Sanierer in der Industrie. Er ist jetzt tätig als selbstständiger Unternehmensberater, Autor, Aufsichtsrat und Hochschullehrer. Internet

http://www.prof-beck.net

\author{
Der Abschied vom alten Chef ist für viele Verbände, \\ Organisationen und Unternehmen ein großer \\ Schritt. Um dabei nicht ins Straucheln zu geraten, \\ sollten einige Ratschläge beherzigt werden.
}

Ein Chefwechsel ist für viele Organisationen von einschneidender Bedeutung. Beschäftigte und Kunden, die externen Partner, die Wettbewerber und natürlich die Aufsichtsgremien - sie alle müssen sich neu orientieren.

Egal, ob Freund oder Feind, sie hatten sich mit dem »Alten « arrangiert. Und jetzt soll das alles nicht mehr gelten? Es ist keine Frage von Glück oder Zufall, ob ein Chefwechsel in einer Organisation erfolgreich verläuft. Nein, Erfolg und Misserfolg haben viele Väter und Mütter, ob sie das wissen und wollen, oder nicht. Hier einige praxisbewährte Tipps:

1. Lassen Sie sich Zeit bei der Aus- wahl. Wenn sie mit dem oder der Neuen zehn gute Jahre haben wollen, dann ist ein Jahr für Suche, Sortierung, Auswahl, Entscheidung und schließlich guter Vorbereitung auf den Start nicht zu viel. Methodische Spielchen müssen nicht sein, aber gründliche Gesprächsgänge durch verschiedene Gesprächspartner sind gut.

2 Klären Sie ganz früh im Wieder- besetzungsprozess, ob Sie eine hausinterne Lösung oder eine externe Lösung bevorzugen. Beides ist möglich, hat aber jeweils auch folgenreiche Konsequenzen. Wenn Ihr Unternehmen immer nur von außen beruft, dann werden die eigenen Leute frustriert. Berufen Sie aber immer nur von innen, dann ist die Innovationskraft in Gefahr. 3. Wenn es einen »Kronprinzen«
3. (oder »Kronprinzessin«) des alten
Chefs gibt, dann klären Sie früh, ob er eine Chance im Besetzungsprozess haben soll. Wenn nein, dann sagen Sie ihm klipp und klar Bescheid! Wenn ja, dann klären Sie ihn rasch darüber auf, ob er "gesetzt « sein wird und einen bevorzugten Startplatz hat, oder ob er sich bescheiden in die Schlange der Bewerber einreihen muss. Feigheit vor dem Kronprinzen schadet der ganzen Firma.

1 Halten Sie den Vorgänger vom 4. Auswahlprozess fern. Schließlich wollen Sie keine Kopie des Bewährten, sondern ein neues, farbiges, frisches Original. Das gilt insbesondere auch für Bewerbervorstellungen und Gehaltsverhandlungen.

5 Halten Sie den Vorgänger vom Auf2. sichtsgremium fern. Er hatte seine Chance als Chef. Wenn er sie genutzt hat, wird er mit allen Ehren abtreten dürfen. Hat er sie verschlafen, dann sollte jetzt auch Ende sein. Und außerdem: Aufsicht ist (heute) etwas ganz anderes als Führung! Man darf heute von einer Führungskraft erwarten, dass sie sich rechtzeitig über ihre Altersaufgaben Gedanken macht, nicht erst am Tag der Verabschiedung.

6. Beziehen Sie die zweite Hierarchieebene in der Endphase der Auswahl mit ein. Sie bestimmt am Ende darüber, ob der neue Chef eine Chance bekommen oder ob er grandios und auf offener Bühne scheitern wird. Der Aufsichtsrat hat bei diesem Spiel nur eine Zuschauerrolle. Er trifft zwar die Ent- 
scheidungen, aber bei der Umsetzung und insbesondere in der Einarbeitungsphase spielt er eine Nebenrolle.

7 Wenn Sie den Vertrag des Neuen - auf fünf, sechs oder sieben Jahre mit der Möglichkeit der Wiederwahl, aber ohne Automatik, befristen, dann sollten Sie ihn so anständig bezahlen, dass er bei verweigerter Wiederwahl nicht in ein soziales Nichts fällt, sondern sich in Ruhe neu orientieren kann. Das bringt Ruhe und langfristige Perspektive in die Führungsarbeit.

Wenn Sie sich für einen Branchen8. fremden entschieden haben, lassen Sie ihn die ersten dummen Fragen ganz woanders stellen, möglichst weit weg, auf keinen Fall in einer sogenannten »befreundeten Einrichtung «, in der sich in einem solchen Falle viele lose Mäuler finden werden. Er sollte erst ins Haus kommen, wenn er mitreden kann. Tun Sie das nicht, dann erhöhen Sie das Risiko seines Scheiterns gewaltig.

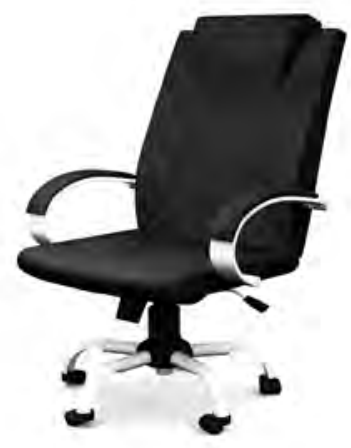

9. Wenn Sie einen Branchenkenner ausgewählt haben, dann stellen Sie sicher, dass er in Ihrem Haus nicht einfach das nochmals wiederholen wird, was ihm woanders (mindestens nach seiner Einschätzung) gelungen ist oder ihm (und hoffentlich auch anderen) zumindest Spaß gemacht hat. Früher ist vorbei und vergangene Heldentaten sind Geschichte. Was Sie jetzt brauchen, ist Zukunft!

10 Investieren Sie mindestens so - viel Zeit und Kraft in die Einarbeitungsphase des Neuen, wie Sie in den Auswahlprozess gesteckt haben. Die wahren Risiken treten erst in der Praxis auf. Wenn Sie hier an der Be- gleitung sparen oder mit Ihrer Intensität nachlassen, dann steigt das Risiko des Scheiterns. Eine Führungsposition wiederzubesetzen kostet - abgesehen vom Imageschaden nach innen und außen - mindestens ein Jahresgehalt. Es wäre zum Beispiel nicht unangemessen, in den ersten Monaten ein monatliches Gespräch mit dem Aufsichtsratsvorsitzenden oder einem dafür beauftragten Aufsichtsratsmitglied zu führen, um frühzeitig Entwicklungen erkennen und gegensteuern zu können.

11. Wenn es in den ersten Monaten - nicht so gut läuft und erste Fragen nach der Eignung des ausgewählten Kandidaten oder nach der Qualität des Auswahlprozesses aufkommen, sollten Sie sich nicht zu unüberlegten Handlungen verführen lassen! Besser ist es, sich diskret zu engagieren, um mögliche Hindernisse aus dem Weg zu räumen.

12. Wenn es dann noch nicht klappt, seien Sie nicht kleinlich! Es ist lächerlich, einer zur Kündigung anstehenden Führungskraft dann mit Kleinigkeiten wie einem unbezahlten Kaffee, privaten Anrufen vom Mobiltelefon oder einigen Euro Bewirtungsaufwendungen zu kommen, um die Abgangskosten vermeintlich zu senken. Das gibt eher einen tiefen Blick auf die Akteure frei und macht einen schlechten Eindruck.

13.

Wenn es aber klappt und alles im Lot ist, dann dürfen Sie sich ganz zu Recht freuen! Sie haben einen wichtigen Beitrag zur Zukunftssicherung des Unternehmens geleistet. Respekt! Jetzt sollten Sie sich aber nicht zurücklehnen. Aufsicht ist eine permanente Aufgabe, bei der die Schwerpunkte »Kontrolle « und »Förderung und Begleitung « beide ihre Berechtigung haben. Und wenn Ihre Führungskräfte fröhlich bei der Arbeit sind, dann macht auch die Aufsichtsratstätigkeit mehr Freude.

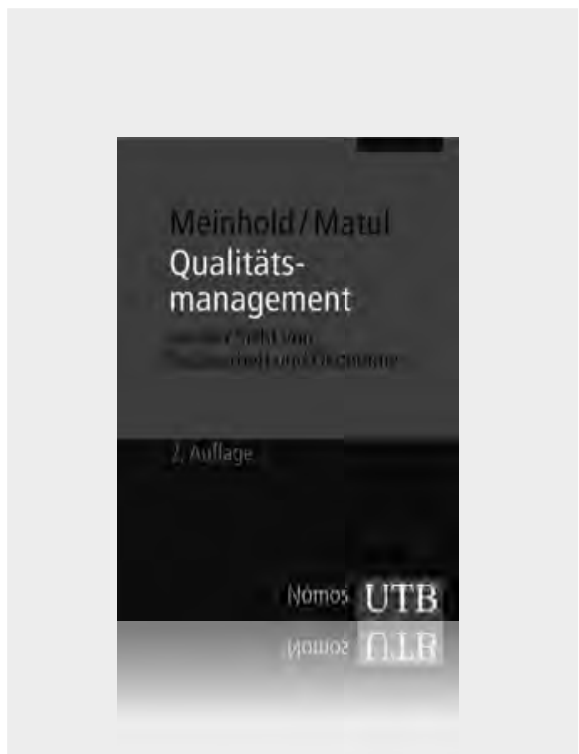

\section{Qualitätsmanagement}

aus der Sicht von Sozialarbeit und Ökonomie

Von Prof. Dr. Marianne Meinhold und Dr. Christian Matul

2. überarbeite und aktualisierte 2011, 177 S., brosch., $38 \mathrm{Abb}, 18,90 €$

ISBN 978-3-8252-3568-0

Im vorliegenden Band werden Möglichkeiten zur Integration sozialer und ökonomischer Sichtweisen beim Qualitätsmanagement aufgezeigt. Anhand von zahlreichen Beispielen können die Leser die einzelnen Schritte zu einer dialogischen Qualitätsentwicklung nachvollziehen, ohne fachfremde bürokratische Forderungen erfüllen zu müssen.

www.nomos-shop.de/13762

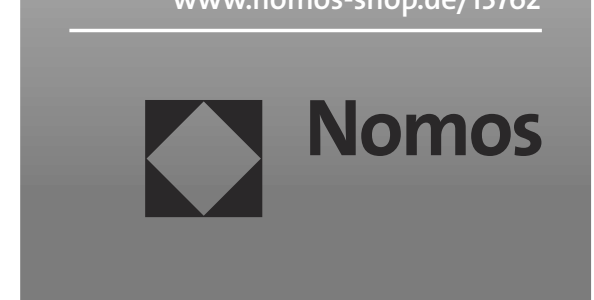

